\title{
Preservation of $t$-norm and $t$-conorm based properties of fuzzy relations during aggregation process
}

\author{
Urszula Dudziak \\ Institute of Mathematics, University of Rzeszów, Al. Rejtana 16 C, 35-959 Reszów
}

\begin{abstract}
In the paper aggregations of fuzzy relations using functions of $n$ variables are considered. After recalling properties of fuzzy relations, aggregation functions which preserve: reflexivity, irreflexivity, $T$-asymmetry, $T$-antisymmetry, symmetry, $S$-connectedness, $T$-transitivity, negative $S$ transitivity, $T$-S-semitransitivity and $T$-S-Ferrers property of fuzzy relations, where $T$ is a $t$-norm and $S$ is a $t$-conorm, are examined.
\end{abstract}

Keywords: Fuzzy relations, properties of fuzzy relations, aggregation functions

\section{Introduction}

Fuzzy relations are often considered in the context of preservation of their properties in the aggregation process (cf. [3], [4], [9], [11], [14] [15], [17]) which is due to the possible applications, e.g. in multicriteria decision making problems. There are diverse types of fuzzy relation properties. We especially take into account the ones which are based on triangular norms and triangular conorms ([9], Chapter 2.5), i.e. $T$-asymmetry, $T$ antisymmetry, $S$-connectedness, $T$-transitivity, negative $S$-transitivity, $T$-S-semitransitivity and $T$-SFerrers property of fuzzy relations, where $T$ is a $t$-norm and $S$ is a $t$-conorm.

In this contribution, we concentrate on a fixed property of fuzzy relations and characterize all aggregation functions preserving this property. Moreover, the aim of this paper is to give characterizations, under the weakest assumptions on functions used for aggregation procedure. Therefore, we start with an arbitrary $n$-ary function (not necessarily aggregation function).

In this paper, we try to present necessary and sufficient conditions for $n$-argument functions to preserve fixed properties of fuzzy relations. Firstly (Section 2), we give basic definitions concerning $n$ ary functions in $[0,1]$, domination between functions and useful notions concerning fuzzy relations. Next, (Section 3), we present theorems for the considered properties of fuzzy relations in the aggregation process.

\section{Basic definitions}

Here we present properties of aggregation functions, the concept of domination and the properties of fuzzy relations considered in this paper.

\subsection{Aggregation functions}

We recall some information about functions in the interval $[0,1]$.

Definition 1 ([2]). Let $n \geqslant 2$. A function $F:[0,1]^{n} \rightarrow[0,1]$ is called an aggregation function if it is increasing in each of its arguments and fulfils the boundary conditions

$$
F(0, \ldots, 0)=0, \quad F(1, \ldots, 1)=1 .
$$

There are many examples of aggregation functions. We present these examples, which will be used in the sequel (cf. [2], [6], [15]).

Example 1. Let $t_{1}, \ldots, t_{n}, w_{1}, \ldots, w_{n} \in[0,1]$. Aggregation functions are:

- the weighted minimum

$F\left(t_{1}, \ldots, t_{n}\right)=\min _{1 \leqslant k \leqslant n} \max \left(1-w_{k}, t_{k}\right), \max _{1 \leqslant k \leqslant n} w_{k}=1$,

- the weighted maximum

$F\left(t_{1}, \ldots, t_{n}\right)=\max _{1 \leqslant k \leqslant n} \min \left(w_{k}, t_{k}\right), \max _{1 \leqslant k \leqslant n} w_{k}=1$,

- the projections

$$
P_{k}\left(t_{1}, \ldots, t_{n}\right)=t_{k}, \quad k \in\{1, \ldots, n\},
$$

- the median value

$$
\operatorname{med}\left(t_{1}, \ldots, t_{n}\right)=\left\{\begin{array}{ll}
\frac{s_{k}+s_{k+1}}{2}, & \text { for } n=2 k \\
s_{k+1}, & \text { for } n=2 k+1
\end{array},\right.
$$

where $\left(s_{1}, \ldots, s_{n}\right)$ is the increasingly ordered sequence of the values $t_{1}, \ldots, t_{n}$, i.e. $s_{1} \leqslant \ldots \leqslant s_{n}$.

- the quasi-linear means

$$
F\left(t_{1}, \ldots, t_{n}\right)=\varphi^{-1}\left(\sum_{k=1}^{n} w_{k} \varphi\left(t_{k}\right)\right),
$$

where $\varphi:[0,1] \rightarrow \mathbb{R}$ is an increasing bijection and the weights $w_{k}>0, k=1, \ldots, n$, fulfil the condition $\sum_{k=1}^{n} w_{k}=1$, 
- the weighted arithmetic mean

$$
F\left(t_{1}, \ldots, t_{n}\right)=\sum_{k=1}^{n} w_{k} t_{k},
$$

which we get if $f(t)=t$ in the formula for a quasilinear mean,

- the weighted geometric mean

$$
F\left(t_{1}, \ldots, t_{n}\right)=\prod_{k=1}^{n} t_{k}^{w_{k}}
$$

which we get if $f(t)=\log t$ in the formula for a quasi-linear mean.

In our further considerations we will need the following concepts.

Definition 2. Let $n \in \mathbb{N}$. We say that a function $F:[0,1]^{n} \rightarrow[0,1]:$

- has a zero element $z \in[0,1]$ (cf. [2], Definition 10) if for each $k \in\{1, \ldots, n\}$ and each $x_{1}, \ldots, x_{k-1}, x_{k+1}, \ldots, x_{n} \in[0,1]$ one has

$$
F\left(x_{1}, \ldots, x_{k-1}, z, x_{k+1}, \ldots, x_{n}\right)=z,
$$

- is without zero divisors if

$$
\underset{x_{1}, \ldots, x_{n} \in[0,1]}{\forall}\left(F\left(x_{1}, \ldots, x_{n}\right)=z \Rightarrow\left(\underset{1 \leqslant k \leqslant n}{\exists} x_{k}=z\right)\right) .
$$

Definition 3 ([12], p. 4). A triangular norm $T:[0,1]^{2} \rightarrow[0,1]$ (triangular conorm $S:[0,1]^{2} \rightarrow$ $[0,1])$ is an arbitrary associative, commutative function having a neutral element $e=1(e=0)$, which is increasing in each of its arguments.

Let us observe that triangular norms (conorms) are aggregation functions for $n=2$. Directly from the definition of a triangular norm (conorm) we obtain a useful property.

Corollary 1. A triangular norm (conorm) has a zero element $z=0(z=1)$.

In our further considerations we will use the abbreviation 't-norm' ('t-conorm') when speaking of a triangular norm (a triangular conorm).

Example 2 ([12], pp. 4, 11). The four well-known examples of t-norms $T$ and corresponding t-conorms $S$ are:

$$
\begin{gathered}
T_{M}(s, t)=\min (s, t), \quad T_{P}(s, t)=s t, \\
S_{M}(s, t)=\max (s, t), \quad S_{P}(s, t)=s+t-s t, \\
T_{L}(s, t)=\max (s+t-1,0), \\
S_{L}(s, t)=\min (s+t, 1), \\
T_{D}(s, t)= \begin{cases}s, & t=1 \\
t, & s=1 \\
0, & \text { otherwise }\end{cases} \\
S_{D}(s, t)= \begin{cases}s, & t=0 \\
t, & s=0 \\
1, & \text { otherwise }\end{cases}
\end{gathered}
$$

for $s, t \in[0,1]$.
Lemma 1 (cf. [12], pp. 6-7). For arbitrary t-norm $T$ and $t$-conorm $S$ one has

$$
T_{D} \leqslant T \leqslant T_{M}, \quad T_{D} \leqslant T_{L} \leqslant T_{P} \leqslant T_{M} .
$$

Moreover

$$
S_{M} \leqslant S \leqslant S_{D}, \quad S_{D} \geqslant S_{L} \geqslant S_{P} \geqslant S_{M} .
$$

Definition 4 ([12], p. 28). A strict t-norm $T$ : $[0,1]^{2} \rightarrow[0,1]$ is a t-norm which is continuous and strictly increasing in $(0,1]^{2}$.

Theorem 1 ([9], p. 11). Any strict t-norm $T$ is a function isomorphic to the product $t$-norm $T_{P}$, i.e.

$T\left(x_{1}, x_{2}\right)=\varphi^{-1}\left(T_{P}\left(\varphi\left(x_{1}\right), \varphi\left(x_{2}\right)\right)\right), \quad x_{1}, x_{2} \in[0,1]$, where $\varphi:[0,1] \rightarrow[0,1]$ is an increasing bijection.

Corollary 2. Triangular norms: $\min , T_{P}$, strict t-norms are functions without zero divisors.

In our further considerations we will need the concept of a dual function and a self-dual function.

Definition 5 (cf. [2], p.31). Let $F:[0,1]^{n} \rightarrow[0,1]$. A function $F^{d}$ is called a dual function to $F$, if for all $x_{1}, \ldots, x_{n} \in[0,1]$

$$
F^{d}\left(x_{1}, \ldots, x_{n}\right)=1-F\left(1-x_{1}, \ldots, 1-x_{n}\right) .
$$

$F$ is called a self-dual function, if it holds $F=F^{d}$.

Example 3 ([2], p.31, [18]). Pairs of dual functions are for example: $T_{M}$ and $S_{M}, T_{L}$ and $S_{L}$, $T_{P}$ and $S_{P}, T_{D}$ and $S_{D}$. In general, $t$-norms are dual functions to $t$-conorms and vice versa. The concept of self-duality is especially developed for aggregation functions. Interesting properties and characterizations of self-dual aggregation functions one can find in [18]. The weighted arithmetic mean, the median and all quasi-linear means for which $f:[0,1] \rightarrow[0,1]$ fulfils $f(1-x)=1-f(x)$, are self-dual aggregation functions. Any self-dual and commutative binary aggregation function $F$ satisfies $F(x, 1-x)=\frac{1}{2}$ for all $x \in[0,1]$, so neither $t$-norms nor $t$-conorms are self-dual.

\subsection{Domination}

Definition 6 (cf. [17], Definition 2.5). Let $m, n \in$ $\mathbb{N}$. A function $F:[0,1]^{m} \rightarrow[0,1]$ dominates function $G:[0,1]^{n} \rightarrow[0,1](F \gg G)$ if for an arbitrary matrix $\left[a_{i k}\right]=A \in[0,1]^{m \times n}$ the following inequality holds

$$
\begin{gathered}
F\left(G\left(a_{11}, \ldots, a_{1 n}\right), \ldots, G\left(a_{m 1}, \ldots, a_{m n}\right)\right) \geqslant \\
G\left(F\left(a_{11}, \ldots, a_{m 1}\right), \ldots, F\left(a_{1 n}, \ldots, a_{m n}\right)\right) .
\end{gathered}
$$

There exist a characterization theorem of all functions which dominate minimum (similarly for a drastic $t$-norm [17]). 
Theorem 2 (cf. [17], Proposition 5.1). A function $F:[0,1]^{n} \rightarrow[0,1]$, which is increasing in each of its arguments dominates minimum if and only if for each $t_{1}, \ldots, t_{n} \in[0,1]$

$$
F\left(t_{1}, \ldots, t_{n}\right)=\min \left(f_{1}\left(t_{1}\right), \ldots, f_{n}\left(t_{n}\right)\right),
$$

where $f_{k}:[0,1] \rightarrow[0,1]$ is increasing with $k=$ $1, \ldots, n$.

Example 4. There are a few useful examples of functions which fulfil conditions of Theorem 2:

if $f_{k}(t)=t, k=1, \ldots, n$, then $F=\min$,

if for a certain $k \in\{1, \ldots, n\}$, function $f_{k}(t)=t$ and $f_{i}(t)=1$ for $i \neq k$, then $F=P_{k}$ - projections,

if $f_{k}(t)=\max \left(1-v_{k}, t\right), v_{k} \in[0,1], k=1, \ldots, n$, $\max _{1 \leqslant k \leqslant n} v_{k}=1$, then $F$ is the weighted minimum.

Here we present some examples of domination between functions.

Example 5 (cf. [8], [17]). The weighted geometric mean dominates t-norm $T_{P}$. The weighted arithmetic mean dominates t-norm $T_{L}$. The function

$$
F\left(t_{1}, \ldots, t_{n}\right)=\frac{p}{n} \sum_{k=1}^{n} t_{k}+(1-p) \min _{1 \leqslant k \leqslant n} t_{k}
$$

dominates $T_{L}$, where $p \in(0,1)$. The weighted minimum dominates every t-norm $T$. Let us consider projections $P_{k}$. Then $F \gg P_{k}$ and $P_{k} \gg F$ for any function $F:[0,1]^{n} \rightarrow[0,1]$ (see $\left.[5]\right)$.

We recall here some results on domination in the family of $t$-norms and $t$-conorms.

Theorem 3 (cf. [17], p.16). Let $T$ be a t-norm, $S$ at-conorm. Then

$$
T \gg T, \quad T_{M} \gg T \gg T_{D},
$$

$$
T_{D} \gg T \Leftrightarrow T=T_{D}, \quad T \gg T_{M} \Leftrightarrow T=T_{M} .
$$

Dually we obtain

$$
\begin{gathered}
S \gg S, \quad S_{D} \gg S \gg S_{M}, \\
S \gg S_{D} \Leftrightarrow S=S_{D}, \quad S_{M} \gg S \Leftrightarrow S=S_{M} .
\end{gathered}
$$

Lemma 2 ([7]). Let $F:[0,1]^{n} \rightarrow[0,1], F$ : $[0,1]^{n} \rightarrow[0,1]$. If $F \gg G$, then $G^{d} \gg F^{d}$.

Corollary 3. Let $F:[0,1]^{n} \rightarrow[0,1]$, and $T$ be a $t$-norm and $S$ be a t-conorm. If $F \gg T$, then $S \gg F^{d}$.

Example 6. By Example 5 and Corollary 3 we see that the weighted arithmetic mean are dominated by $S_{L}$ and the weighted maximum is dominated by any $t$-conorm $S$. Moreover, it is easy to verify that the function

$$
F\left(t_{1}, \ldots, t_{n}\right)=\frac{p}{n} \sum_{k=1}^{n} t_{k}+(1-p) \max _{1 \leqslant k \leqslant n} t_{k}
$$

is dominated by $S_{L}$, where $p \in(0,1)$. By Theorem 2 and Corollary 3 a function $F:[0,1]^{n} \rightarrow[0,1]$, which is increasing in each of its arguments is dominated by maximum if and only if for each $t_{1}, \ldots, t_{n} \in[0,1]$

$$
F\left(t_{1}, \ldots, t_{n}\right)=\max \left(f_{1}\left(t_{1}\right), \ldots, f_{n}\left(t_{n}\right)\right),
$$

where $f_{k}:[0,1] \rightarrow[0,1]$ is increasing with $k=$ $1, \ldots, n$. Examples of such functions are: if $f_{k}(t)=t, k=1, \ldots, n$, then $F=\max$,

if for a certain $k \in\{1, \ldots, n\}$, function $f_{k}(t)=t$ and $f_{i}(t)=1$ for $i \neq k$, then $F=P_{k}$ - projections, if $f_{k}(t)=\min \left(v_{k}, t\right), v_{k} \in[0,1], k=1, \ldots, n$, $\max _{1 \leqslant k \leqslant n} v_{k}=1$, then $F$ is the weighted maximum.

For further results on domination, specially domination between t-norms, we recommend [12] (pp.152-156), [13], [19], we recall only a few of them which will be useful later on.

\subsection{Fuzzy relations}

We recall basic properties of fuzzy relations.

Definition 7 ([20]). A fuzzy relation on a set $X \neq \emptyset$ is an arbitrary function $R: X \times X \rightarrow[0,1]$. The family of all fuzzy relations on $X$ is denoted by $F R(X)$.

Remark 1. If card $X=n, X=\left\{x_{1}, \ldots, x_{n}\right\}$, then $R \in F R(X)$ may be presented by a matrix $R=\left[r_{i k}\right]$, where $r_{i k}=R\left(x_{i}, x_{k}\right), i, k=1, \ldots, n$.

Definition 8 (cf. [20]). Let $*:[0,1]^{2} \rightarrow[0,1]$. A sup-*-composition of relations $R, W \in F R(X)$ is the relation $(R \circledast W) \in F R(X)$ such that for any $(x, z) \in X \times X$

$$
(R \circledast W)(x, z)=\sup _{y \in X} R(x, y) * W(y, z) .
$$

An inf-*-composition of relations $R, W \in F R(X)$ is the relation $\left(R \circledast^{\prime} W\right) \in F R(X)$ such that for any $(x, z) \in X \times X$

$$
\left(R \circledast{ }^{\prime} W\right)(x, z)=\inf _{y \in X} R(x, y) * W(y, z) .
$$

Next we present properties of fuzzy relations, which will be discussed in our further considerations ([1], p. 38, [9], Chapter 2.5, cf. [10], p. 445).

Definition 9. Let $T$ be a $t$-norm and $S$ be a $t$-conorm. Relation $R \in F R(X)$ is:

- reflexive, if $\underset{x \in X}{\forall} R(x, x)=1$,

- irreflexive, if $\underset{x \in X}{\forall} R(x, x)=0$,

- symmetric, if $\underset{x, y \in X}{\forall} R(x, y)=R(y, x)$,

- T-asymmetric, if $\underset{x, y \in X}{\forall} T(R(x, y), R(y, x))=0$,

- T-antisymmetric, if $\underset{x, y, x \neq y \in X}{\forall} T(R(x, y), R(y, x))=0$,

- totally S-connected, if $\underset{x, y \in X}{\forall} S(R(x, y), R(y, x))=1$, 
- S-connected, if $\underset{x, y, x \neq y \in X}{\forall} S(R(x, y), R(y, x))=1$,

- T-transitive, if

$\underset{x, z \in X}{\forall} T(R(x, y), R(y, z)) \leqslant R(x, z)$,

- negatively $\mathrm{S}$-transitive, if

$\underset{y, z \in X}{\forall} S(R(x, y), R(y, z)) \geqslant R(x, z)$,

- T-S-Ferrers, if

$\underset{y, z, w \in X}{\forall} T(R(x, y), R(z, w)) \leqslant S(R(x, w), R(z, y))$,

- T-S-semitransitive, if

$\underset{x, y, z, w \in X}{\forall} T(R(x, w), R(w, y)) \leqslant S(R(x, z), R(z, y))$.

In the above definition a $t$-norm takes place of a minimum and a $t$-conorm takes place of a maximum in the standard properties introduced for fuzzy relations by L.A. Zadeh [21] (for the adequate properties of crisp relations see [16]).

With the use of $n$-argument function $F, n \in$ $\mathbb{N}, n \geqslant 2$, we aggregate given fuzzy relations $R_{1}, \ldots, R_{n}$.

Definition 10 ([17], Definition 2.4). Let $F$ : $[0,1]^{n} \rightarrow[0,1], R_{1}, \ldots, R_{n} \in F R(X)$. An aggregated fuzzy relation $R_{F} \in F R(X)$ is described by the formula

$$
R_{F}(x, y)=F\left(R_{1}(x, y), \ldots, R_{n}(x, y)\right), \quad x, y \in X .
$$

A function $F$ preserves a property of fuzzy relations if for every $R_{1}, \ldots, R_{n} \in F R(X)$ having this property, $R_{F}$ also has this property.

We recall here the concept of the complement $R^{\prime}$ of a fuzzy relation $R$, i.e.

$$
R^{\prime}(x, y)=1-R(x, y), x, y \in[0,1]
$$

Lemma 3. A fuzzy relation $R$ is T-asymmetric (T-antisymmetric, $T$-transitive), if and only if its complement $R^{\prime}$ is totally $S$-connected ( $S$-connected, negatively $S$-transitive).

In virtue of this lemma conditions for aggregated totally $S$-connected, $S$-connected and negatively $S$ transitive fuzzy relations can be obtained by negation of conditions considered above for aggregated $T$-asymmetric, $T$-antisymmetric and $T$-transitive fuzzy relations, respectively.

\section{Preservation of fuzzy relation properties}

In this section we present the weakest assumptions on functions to preserve fuzzy relation properties. Aggregation functions (cf. Definition 1) are given as examples. We recall here the results from [8] concerning preservation of reflexivity and symmetry.

Theorem 4. Let $R_{1}, \ldots, R_{n} \in F R(X)$ be reflexive (respectively irreflexive). The relation $R_{F}$ is reflexive (respectively irreflexive), if and only if the function $F$ satisfies the condition $F(1, \ldots, 1)=1$ (respectively $F(0, \ldots, 0)=0)$.
Any aggregation function and any idempotent function $F$, i.e. function which fulfils $F(t, \ldots, t)=$ $t$, for $t \in[0,1]$ preserves reflexivity and irreflexivity of fuzzy relations.

Theorem 5. Let $R_{1}, \ldots, R_{n} \in F R(X)$ be symmetric. For every function $F$ the fuzzy relation $R_{F}$ is also symmetric.

Here theorems concerning preservation of $t$-norm and $t$-conorm based fuzzy relation properties will be presented.

Theorem 6. Let card $X \geqslant 2, T$ be a t-norm without zero divisors. A function $F$ preserves $T$-asymmetry (T-antisymmetry) if and only if it satisfies the following condition for all $s, t \in[0,1]^{n}$

$$
\underset{1 \leqslant k \leqslant n}{\forall} \min \left(s_{k}, t_{k}\right)=0 \Rightarrow \min (F(s), F(t))=0 .
$$

Proof. Let $F$ fulfil (5) for all $s, t \in[0,1]^{n}, x, y \in$ $X$. If $R_{1}, \ldots, R_{n} \in F R(X)$ are $T$-asymmetric, then using substitution $s_{k}=R_{k}(x, y), t_{k}=R_{k}(y, x)$ for $k=1, \ldots, n$ we see that for a $t$-norm $T$ without zero divisors we get $s_{k}=0$ or $t_{k}=0, k=1, \ldots, n$, as a result

$$
\underset{1 \leqslant k \leqslant n}{\forall} \min \left(s_{k}, t_{k}\right)=0
$$

and the relation $R_{F}$ is $T$-asymmetric by (5) and the assumption on a $t$-norm $T$.

Conversely, let $s, t \in[0,1]^{n}$ fulfil (6). Since card $X \geqslant 2$, then there exist $a, b \in X, a \neq b$. Fuzzy relations

$R_{k}(x, y)= \begin{cases}s_{k}, & \text { if }(x, y)=(a, b) \\ t_{k}, & \text { if }(x, y)=(b, a), \quad k=1, \ldots, n \\ 0, & \text { otherwise }\end{cases}$

are $T$-asymmetric. Thus, the relation $R_{F}$ is also $T$-asymmetric and we get

$$
\begin{gathered}
T(F(s), F(t))= \\
T\left(F\left(R_{1}(a, b), \ldots, R_{n}(a, b)\right),\right. \\
\left.F\left(R_{1}(b, a), \ldots, R_{n}(b, a)\right)\right)=0,
\end{gathered}
$$

so $\min (F(s), F(t))=0$ which proves (5). In the case of $T$-antisymmetric fuzzy relations the proof is similar.

Now we give examples of functions preserving $T$-asymmetry and $T$-antisymmetry. We apply here Corollary 2.

Example 7 ([8]). Let $T$ be a strict $t$-norm. The function $F=$ min preserves $T$-asymmetry ( $T$-antisymmetry). Functions $F$ which has the zero element $z=0$ with respect to certain coordinate, i.e.

$\underset{1 \leqslant k \leqslant n}{\exists} \underset{i \neq k}{\forall} \underset{t_{i} \in[0,1]}{\forall} F\left(t_{1}, \ldots, t_{k-1}, 0, t_{k+1}, \ldots, t_{n}\right)=0$ 
fulfil (5), so they preserve $T$-asymmetry ( $T$-antisymmetry). In particular, the weighted geometric mean fulfil (5). As another example we may consider the median function. If a function $F$ fulfils the condition

$$
\underset{t \in[0,1]^{n}}{\forall} \operatorname{card}\left\{k: t_{k}=0\right\}>\frac{n}{2} \Rightarrow F(t)=0,
$$

then we also get (5) (e.g. the median fulfils this condition). However, the above condition is not necessary for (5), because it does not cover the projections which preserve $T$-asymmetry ( $T$-antisymmetry).

Similarly as in Theorem 6 we get

Theorem 7. Let card $X \geqslant 2, S$ be a t-conorm without zero divisors. A function $F$ preserves total $S$-connectedness ( $S$-connectedness) if and only if it satisfies the following condition for all $s, t \in[0,1]^{n}$

$$
\underset{1 \leqslant k \leqslant n}{\forall} \max \left(s_{k}, t_{k}\right)=1 \Rightarrow \max (F(s), F(t))=1 .
$$

Proof. Let $F$ fulfil (8) for all $s, t \in[0,1]^{n}, x, y \in X$. If $R_{1}, \ldots, R_{n} \in F R(X)$ are totally $S$-connected, then using substitution $s_{k}=R_{k}(x, y), t_{k}=R_{k}(y, x)$ for $k=1, \ldots, n$ we see that for a $t$-conorm $S$ without zero divisors we get $s_{k}=1$ or $t_{k}=1$, $k=1, \ldots, n$, as a result

$$
\underset{1 \leqslant k \leqslant n}{\forall} \max \left(s_{k}, t_{k}\right)=1
$$

and the relation $R_{F}$ is totally $S$-connected by (8) and the assumption on a $t$-conorm $S$.

Conversely, let $s, t \in[0,1]^{n}$ fulfil (9). Since card $X \geqslant 2$, then there exist $a, b \in X$ such that $a \neq b$. Fuzzy relations

$$
R_{k}(x, y)= \begin{cases}s_{k}, & \text { if }(x, y)=(a, b) \\ t_{k}, & \text { if }(x, y)=(b, a), \quad k=1, \ldots, n \\ 1, & \text { otherwise }\end{cases}
$$

are totally $S$-connected. Thus, the relation $R_{F}$ is also totally $S$-connected and we get

$$
\begin{gathered}
S(F(s), F(t))= \\
S\left(F\left(R_{1}(a, b), \ldots, R_{n}(a, b)\right),\right. \\
\left.F\left(R_{1}(b, a), \ldots, R_{n}(b, a)\right)\right)=1,
\end{gathered}
$$

so $\max (F(s), F(t))=1$ which proves (8). In the case of $S$-connected fuzzy relations the proof is analogous.

Example 8. Examples of functions fulfilling (8) for all $s, t \in[0,1]^{n}$ are $F=\max , F=$ med or functions $F$ with the zero element $z=1$ with respect to a certain coordinate, i.e.

$$
\underset{1 \leqslant k \leqslant n}{\exists} \underset{i \neq k}{\forall} \underset{t_{i} \in[0,1]}{\forall} F\left(t_{1}, \ldots, t_{k-1}, 1, t_{k+1}, \ldots, t_{n}\right)=1 .
$$

The dual property for (7) have the form

$$
\underset{t \in[0,1]^{n}}{\forall} \operatorname{card}\left\{k: t_{k}=1\right\}>\frac{n}{2} \Rightarrow F(t)=1 .
$$

We will give theorems (Theorems 8, 9) providing a necessary and a sufficient condition for the preservation of $T$-transitivity (cf. also [17]). This are modified versions of theorems obtained in [8] for more general class of $*$-transitive fuzzy relations, where $*:[0,1]^{2} \rightarrow[0,1]$.

Theorem 8. Let card $X \geqslant 3$. If a function $F:[0,1]^{n} \rightarrow[0,1]$ preserves $T$-transitivity, then it dominates $T(F \gg T)$, it means that for all $\left(s_{1}, \ldots, s_{n}\right),\left(t_{1}, \ldots, t_{n}\right) \in[0,1]^{n}$

$$
\begin{gathered}
F\left(T\left(s_{1}, t_{1}\right), \ldots, T\left(s_{n}, t_{n}\right)\right) \geqslant \\
T\left(F\left(s_{1}, \ldots, s_{n}\right), F\left(t_{1}, \ldots, t_{n}\right)\right) .
\end{gathered}
$$

Theorem 9. If a function $F:[0,1]^{n} \rightarrow[0,1]$, which is increasing in each of its arguments fulfils $F \gg T$, then it preserves T-transitivity.

Example 9 (cf. [17], p. 30). Each quasi-linear mean dominates $T_{D}$. Moreover, for $n=2$ arbitrary t-norm $F=T$ dominates $T_{D}$. By Theorem 2 and Examples $4-5$ we see that the minimum, the weighted minimum and the projections dominate min. The weighted geometric mean preserves $T_{P}$-transitivity, the weighted arithmetic mean preserves $T_{L}$-transitivity, the minimum preserves $T$-transitivity with arbitrary t-norm $T$. The function $F$ described by the formula (1) preserves $T_{L}$-transitivity.

In Theorems 8 and 9 we present respectively necessary and sufficient conditions for the preservation of $T$-transitivity. We give separate results to point out the adequate assumptions for each theorem. Dually to the result for $T$-transitivity (see [8]) we may obtain the statement for negative $S$-transitivity. To prove it, we will need the property connected with the extension of a relation $R_{0} \in F R(A)$ to the relation $R \in F R(X)$, where $A \subset X, A \neq \emptyset$.

Lemma 4. Let $R_{0}, W_{0} \in F R(A), \emptyset \neq A \subset X$, $R, W \in F R(X)$ be of the form

$$
\begin{aligned}
& R(x, y)=\left\{\begin{array}{ll}
R_{0}(x, y), & x, y \in A \\
1, & \text { otherwise }
\end{array},\right. \\
& W(x, y)=\left\{\begin{array}{ll}
W_{0}(x, y), & x, y \in A \\
1, & \text { otherwise }
\end{array},\right.
\end{aligned}
$$

respectively. If an operation $*:[0,1]^{2} \rightarrow[0,1]$ has the zero element $z=1$, then

$\left(R \circledast{ }^{\prime} W\right)(x, y)=\left\{\begin{array}{ll}\left(R_{0} \circledast{ }^{\prime} W_{0}\right)(x, y), & x, y \in A \\ 1, & \text { otherwise }\end{array}\right.$.

Proof. Let $x, y \in X, B=X \backslash A$. By the associativity of the infimum on $[0,1]$ we may consider the following cases:

if $(x, y) \in A \times A$, then

$$
\left.(R \circledast)^{\prime} W\right)(x, y)=\inf _{w \in X} R(x, w) * W(w, y)=
$$


$\min \left(\inf _{w \in A} R(x, w) * W(w, y), \inf _{w \in B} R(x, w) * W(w, y)\right)=$ $\min \left(\inf _{w \in A} R_{0}(x, w) * W_{0}(w, y), \inf _{w \in B} 1 * 1\right)=$ $\min \left(\left(R_{0} \circledast{ }^{\prime} W_{0}\right)(x, y), 1\right)=\left(R_{0} \circledast{ }^{\prime} W_{0}\right)(x, y)$, if $(x, y) \in A \times B$, then

$$
\left(R \circledast{ }^{\prime} W\right)(x, y)=\inf _{w \in X} R(x, w) * W(w, y)=
$$

$\min \left(\inf _{w \in A} R(x, w) * W(w, y), \inf _{w \in B} R(x, w) * W(w, y)\right)=$ $\min \left(\inf _{w \in A} R_{0}(x, w) * 1, \inf _{w \in B} 1 * 1\right)=\min (1,1)=1$,

if $(x, y) \in B \times A$, then

$$
\left(R \circledast{ }^{\prime} W\right)(x, y)=\inf _{w \in X} R(x, w) * W(w, y)=
$$

$\min \left(\inf _{w \in A} R(x, w) * W(w, y), \inf _{w \in B} R(x, w) * W(w, y)\right)=$ $\min \left(\inf _{w \in A} 1 * W_{0}(w, y), \inf _{w \in B} 1 * 1\right)=\min (1,1)=1$, if $(x, y) \in B \times B$, then

$$
\begin{gathered}
\left(R \circledast{ }^{\prime} W\right)(x, y)=\inf _{w \in X} R(x, w) * W(w, y)= \\
\inf _{w \in X}(1 * 1)=1 .
\end{gathered}
$$

As a result $R \circledast{ }^{\prime} W$ is of the form (12).

Lemma 5. Let $*$ be a t-conorm. A relation $R \in$ $F R(X)$ is negatively $*$-transitive if and only if

$$
R \circledast)^{\prime} R \geqslant R .
$$

Proof. Let $R \in F R(X)$. Directly by (4) and definition of negative $S$-transitivity we get

$$
\begin{aligned}
R \circledast{ }^{\prime} R & \geqslant R \Leftrightarrow \underset{x, z \in X}{\forall} \inf _{y \in X} R(x, y) * R(y, z) \geqslant R(x, z) \\
& \Leftrightarrow \underset{x, y, z \in X}{\forall} R(x, y) * R(y, z) \geqslant R(x, z),
\end{aligned}
$$

which means that $R$ is negatively $*$-transitive for a $t$-conorm $*$.

Lemma 6. Let $S$ be a t-conorm, $A \subset X, A \neq \emptyset$. If relation $R_{0} \in F R(A)$ is negatively $S$-transitive in $A$, then relation $R \in F R(X)$ described by (10) is negatively $S$-transitive in $X$.

Proof. Let $(x, y) \in A \times A$. By Lemma 4 , Lemma 5 and by negative $S$-transitivity of relation $R_{0}$ in $A$ we get for any $x, y \in A$

$\left(R \circledast{ }^{\prime} R\right)(x, y)=\left(R_{0} \circledast{ }^{\prime} R_{0}\right)(x, y) \geqslant R_{0}(x, y)=R(x, y)$.

If $(x, y) \in X \times X \backslash A \times A$, then $\left(R \circledast{ }^{\prime} R\right)(x, y)=$ $1 \geqslant R(x, y)$. As a result relation $R$ is negatively $S$-transitive in $X$.
Theorem 10. Let card $X \geqslant 3$. An increasing in each of its arguments function $F:[0,1]^{n} \rightarrow$ $[0,1]$ preserves negative $S$-transitivity if and only if $S \gg F$, it means that for any $\left(s_{1}, \ldots, s_{n}\right)$, $\left(t_{1}, \ldots, t_{n}\right) \in[0,1]^{n}$ the following inequality holds

$$
\begin{gathered}
S\left(F\left(s_{1}, \ldots, s_{n}\right), F\left(t_{1}, \ldots, t_{n}\right)\right) \geqslant \\
F\left(S\left(s_{1}, t_{1}\right), \ldots, S\left(s_{n}, t_{n}\right)\right) .
\end{gathered}
$$

Proof. Let us fix $s_{k}, t_{k} \in[0,1], k=1, \ldots, n$ and take $u, v, w \in X$. By assumption that card $X \geqslant 3$, we may write $u \neq v, v \neq w, u \neq w$. We also denote $A=\{u, v, w\}$. Firstly, we will create fuzzy relations $W_{k}, k=1, \ldots, n$ in the set $A$ described by the matrices

$$
W_{k}=\left[\begin{array}{ccc}
1 & s_{k} & S\left(s_{k}, t_{k}\right) \\
1 & 1 & t_{k} \\
1 & 1 & 1
\end{array}\right], k=1, \ldots, n .
$$

In other words

$W_{k}(u, v)=s_{k}, W_{k}(v, w)=t_{k}, W_{k}(u, w)=S\left(s_{k}, t_{k}\right)$.

By Lemma 5 relations $W_{k}$ are negatively $S$ transitive in $A$ because

$W_{k} \circledast{ }^{\prime} W_{k}=\left[\begin{array}{ccc}1 & 1 & S\left(s_{k}, t_{k}\right) \\ 1 & 1 & 1 \\ 1 & 1 & 1\end{array}\right] \geqslant W_{k}, \quad k=1, \ldots, n$.

In virtue of Lemma 6 relations $R_{k} \in F R(X), k=$ $1, \ldots, n$,

$$
R_{k}(x, y)= \begin{cases}W_{k}(x, y), & x, y \in A \\ 1, & \text { otherwise }\end{cases}
$$

are negatively $S$-transitive in $X$. By assumption $F$ preserves negative $S$-transitivity of fuzzy relations so relation $R_{F}=F\left(R_{1}, \ldots, R_{n}\right)$ is also negatively $S$-transitive for $x, y, z \in X$, i.e.

$$
\begin{gathered}
S\left(F\left(R_{1}(x, y), \ldots, R_{n}(x, y)\right), F\left(R_{1}(y, z), \ldots, R_{n}(y, z)\right)\right) \\
\geqslant F\left(R_{1}(x, z), \ldots, R_{n}(x, z)\right) .
\end{gathered}
$$

In particular, for elements $u, v, w \in X$ the above inequality is also fulfilled. Thus applying the notations (15) and formula (16) we have

$$
\begin{gathered}
S\left(F\left(s_{1}, \ldots, s_{n}\right), F\left(t_{1}, \ldots, t_{n}\right)\right)= \\
S\left(F\left(R_{1}(u, v), \ldots, R_{n}(u, v)\right), F\left(R_{1}(v, w), \ldots, R_{n}(v, w)\right)\right) \\
\geqslant \\
F\left(R_{1}(u, w), \ldots, R_{n}(u, w)\right)= \\
F\left(S\left(s_{1}, t_{1}\right), \ldots, S\left(s_{n}, t_{n}\right)\right),
\end{gathered}
$$

which means that operation $F$ fulfils (14).

Now, let an increasing operation $F$ fulfil (14), $x, y, z \in X$. If relations $R_{k}$ are negatively $S$-transitive for $k=1, \ldots, n$, then $S\left(R_{k}(x, y), R_{k}(y, z)\right) \geqslant R_{k}(x, z)$. We will prove 
that the relation $R_{F}$ is negatively $S$-transitive. Applying the notations $R_{k}(x, y)=s_{k}, R_{k}(y, z)=t_{k}$, for $k=1, \ldots, n$ we obtain

$$
\begin{gathered}
S\left(R_{F}(x, y), R_{F}(y, z)\right)= \\
S\left(F\left(R_{1}(x, y), \ldots, R_{n}(x, y)\right), F\left(R_{1}(y, z), \ldots, R_{n}(y, z)\right)\right)= \\
S\left(F\left(s_{1}, \ldots, s_{n}\right), F\left(t_{1}, \ldots, t_{n}\right)\right) \geqslant \\
F\left(S\left(s_{1}, t_{1}\right), \ldots, S\left(s_{n}, t_{n}\right)\right)= \\
F\left(S\left(R_{1}(x, y), R_{1}(y, z)\right), \ldots, S\left(R_{n}(x, y), R_{n}(y, z)\right)\right) \geqslant \\
F\left(R_{1}(x, z), \ldots, R_{n}(x, z)\right)=R_{F}(x, z) .
\end{gathered}
$$

As a result operation $F$ preserves negative $S$-transitivity.

Example 10. By Example 6 we see that the weighted maximum preserves negative $S$ transitivity for any $t$-conorm $S$. The weighted arithmetic mean and the functions of the form (2) preserve negative $S_{L}$-transitivity. Moreover, negative $S_{M}$-transitivity (i.e. negative transitivity) is preserved if and only if a function $F$ is of the form

$$
F\left(t_{1}, \ldots, t_{n}\right)=\max \left(f_{1}\left(t_{1}\right), \ldots, f_{n}\left(t_{n}\right)\right),
$$

for each $t_{1}, \ldots, t_{n} \in[0,1]$ and $f_{k}:[0,1] \rightarrow[0,1]$ being increasing with $k=1, \ldots, n$.

Now will consider $T$-S-Ferrers property. The other result for preservation of this property (and also $T$-transitivity) for quasi-arithmetic means one can find in [9], p. 140-141.

Theorem 11. If a function $F:[0,1]^{n} \rightarrow[0,1]$, which is increasing in each of its arguments fulfils $F \gg T$ and $S \gg F$, then it preserves $T$-S-Ferrers property.

Proof. Let an increasing function $F$ fulfil $F \gg T$ and $S \gg F, x, y, u, v \in X$. If relations $R_{k}$ have $T$-S-Ferrers property for $k=1, \ldots, n$, then $T\left(R_{k}(x, y), R_{k}(u, v)\right) \leqslant S\left(R_{k}(x, v), R_{k}(u, y)\right)$. We will prove that the relation $R_{F}$ has $T$-S-Ferrers property. We obtain

$$
\begin{gathered}
T\left(R_{F}(x, y), R_{F}(u, v)\right)= \\
T\left(F\left(R_{1}(x, y), \ldots, R_{n}(x, y)\right), F\left(R_{1}(u, v), \ldots, R_{n}(u, v)\right)\right) \\
\leqslant \\
F\left(T\left(R_{1}(x, y), R_{1}(u, v)\right), \ldots, T\left(R_{n}(x, y), R_{n}(u, v)\right)\right) \leqslant \\
F\left(S\left(R_{1}(x, v), R_{1}(u, y)\right), \ldots, S\left(R_{n}(x, v), R_{n}(u, y)\right)\right) \leqslant \\
S\left(F\left(R_{1}(x, v), \ldots, R_{n}(x, v)\right), F\left(R_{1}(u, y), \ldots, R_{n}(u, y)\right)\right) \\
=S\left(R_{F}(x, v), R_{F}(u, y)\right) .
\end{gathered}
$$

As a result function $F$ preserves $T$-S-Ferrers property.

The next statement gives a sufficient condition for a function $F$ to preserve $T$-S-Ferrers property.
Lemma 7. Let $T$ be a t-norm and $S$ be a corresponding dual t-conorm. If $F:[0,1]^{n} \rightarrow[0,1]$ is a self-dual function, then $F \gg T$ implies $S \gg F$.

Proof. Let $x_{1}, \ldots, x_{n}, y_{1}, \ldots, y_{n} \in[0,1]$. If $F \gg T$ we obtain

$$
\begin{gathered}
F\left(T\left(x_{1}, y_{1}\right), \ldots, T\left(x_{n}, y_{n}\right)\right) \geqslant \\
T\left(F\left(x_{1}, \ldots, x_{n}\right), F\left(y_{1}, \ldots, y_{n}\right)\right) .
\end{gathered}
$$

Since $T(x, y)=1-S(1-x, 1-y)$ for $x, y \in[0,1]$, it follows

$$
\begin{gathered}
F\left(1-S\left(1-x_{1}, 1-y_{1}\right), \ldots, 1-S\left(1-x_{n}, 1-y_{n}\right)\right) \geqslant \\
1-S\left(1-F\left(x_{1}, \ldots, x_{n}\right), 1-F\left(y_{1}, \ldots, y_{n}\right)\right) .
\end{gathered}
$$

As a result

$$
\begin{gathered}
1-F\left(1-S\left(1-x_{1}, 1-y_{1}\right), \ldots, 1-S\left(1-x_{n}, 1-y_{n}\right)\right) \leqslant \\
S\left(1-F\left(x_{1}, \ldots, x_{n}\right), 1-F\left(y_{1}, \ldots, y_{n}\right)\right),
\end{gathered}
$$

and by $F=F^{d}$ we get

$$
\begin{gathered}
F\left(S\left(1-x_{1}, 1-y_{1}\right), \ldots, S\left(1-x_{n}, 1-y_{n}\right)\right) \leqslant \\
S\left(1-F\left(x_{1}, \ldots, x_{n}\right), 1-F\left(y_{1}, \ldots, y_{n}\right)\right) .
\end{gathered}
$$

By substitution $1-x_{i}=a_{i}$ and $1-y_{i}=b_{i}$ for $i=1, \ldots, n$, where $a_{i}, b_{i} \in[0,1]$ and applying again the assumption $F=F^{d}$ we obtain

$$
\begin{gathered}
F\left(S\left(a_{1}, b_{1}\right), \ldots, S\left(a_{n}, b_{n}\right)\right) \leqslant \\
S\left(F\left(a_{1}, \ldots, a_{n}\right), F\left(b_{1}, \ldots, b_{n}\right)\right),
\end{gathered}
$$

which proves that $S \gg F$.

Example 11. By Example 5 we know that the weighted arithmetic mean dominate $T_{L}$ and the weighted arithmetic mean are self-dual aggregation functions (Example 3), so by Lemma 7 we see that the weighted arithmetic mean preserve $T$ - $S$-Ferrers property for $T_{L}$ and $S_{L}$.

Now $T$-S-semitransitivity will be discussed.

Theorem 12. If a function $F:[0,1]^{n} \rightarrow[0,1]$, which is increasing in each of its arguments fulfils $F \gg T$ and $S \gg F$, then it preserves $T-S$ semitransitivity.

Proof. Let an increasing function $F$ fulfil $F \gg$ $T$ and $S \gg F, x, y, u, v \in X$. If relations $R_{k}$ are $T$-S-semitransitive for $k=1, \ldots, n$, then $T\left(R_{k}(x, y), R_{k}(y, v)\right) \leqslant S\left(R_{k}(x, u), R_{k}(u, v)\right)$. We will prove that the relation $R_{F}$ is $T-S$ semitransitive. We obtain

$$
\begin{gathered}
T\left(R_{F}(x, y), R_{F}(y, v)\right)= \\
T\left(F\left(R_{1}(x, y), \ldots, R_{n}(x, y)\right), F\left(R_{1}(y, v), \ldots, R_{n}(y, v)\right)\right) \\
\leqslant \\
F\left(T\left(R_{1}(x, y), R_{1}(y, v)\right), \ldots, T\left(R_{n}(x, y), R_{n}(y, v)\right)\right) \leqslant \\
F\left(S\left(R_{1}(x, u), R_{1}(u, v)\right), \ldots, S\left(R_{n}(x, u), R_{n}(u, v)\right)\right) \leqslant \\
S\left(F\left(R_{1}(x, u), \ldots, R_{n}(x, u)\right), F\left(R_{1}(u, v), \ldots, R_{n}(u, v)\right)\right) \\
=S\left(R_{F}(x, u), R_{F}(u, v)\right) .
\end{gathered}
$$

As a result function $F$ preserves $T$-S-semitransitivity. 
Examples of functions which preserve $T$-S-semitransitivity are the same as for $T$-S-Ferrers property.

Example 12. Conditions given in Theorems 11 and 12 are only the sufficient ones. Let us consider function $F(s, t)=s t$ (so $F=T_{P}$ ) and fuzzy relations presented by the matrices

$$
R_{1}=\left[\begin{array}{ll}
0 & 1 \\
0 & 0
\end{array}\right], \quad R_{2}=\left[\begin{array}{ll}
0 & 0 \\
1 & 0
\end{array}\right] .
$$

Relations $R_{1}, R_{2}$ are min-max-Ferrers ([9], p. 142) and min-max-semitransitive, and $R=F\left(R_{1}, R_{2}\right)$ is both min-max-Ferrers and min-max-semitransitive, where $R \equiv 0$. However, it is not true that $F \gg$ min (the only $t$-norm that dominates minimum is minimum itself, see Theorem 3).

\section{Conclusion}

In this contribution we presented the necessary and sufficient conditions for the preservation of fuzzy relation properties. The considered properties involve triangular norms $T$ and triangular conorms $S$. In the case of $T$-S-Ferrers property and $T$-S-semitransitivity only the sufficient conditions were obtained, but suitable counter-examples showing that the necessity does not hold were provided.

\section{References}

[1] U. Bodenhofer, A Similarity-based Generalization of Fuzzy Orderings, $\mathrm{PhD}$ thesis, Universitätsverlag Rudolf Trauner, Linz, 1999.

[2] T. Calvo, A. Kolesárová, M. Komorníková and R. Mesiar, Aggregation operators: properties, classes and construction methods, In T. Calvo, G. Mayor and R. Mesiar, editors, Aggregation Operators vol. 9\%: Studies in Fuzziness and Soft Computing, pages 3-104, Physica-Verlag, Heildelberg, 2002.

[3] B. De Baets and R. Mesiar, T-partitions, Fuzzy Sets Syst., 97:211-223, Elsevier, 1998.

[4] F. Chiclana, F. Herrera, E. Herrera-Viedma and L. Martínez, A note on the reciprocity in the aggregation of fuzzy preference relations using OWA oprators, Fuzzy Sets Syst., 137:7183, Elsevier, 2003.

[5] J. Drewniak, P. Drygaś and U. Dudziak, Domination between multiplace operations, In O. Hryniewicz, J. Kacprzyk and D. Kuchta, editors, Issues in Soft Computing, Decisions and Operations Research, pages 149-160, EXIT, Warszawa, 2005.

[6] J. Drewniak and U. Dudziak, Aggregations preserving classes of fuzzy relations, Kybernetika, 41(3):265-284, Institute of Information Theory and Automation Academy of Sciences of Czech Republic, 2005.

[7] J. Drewniak and A. Król, On the problem of domination between triangular norms and conorms, Journal of Electrical Engineering, 56(12/s):59-61, Slovak Centre of IEE, 2005.

[8] J. Drewniak and U. Dudziak, Preservation of properties of fuzzy relations during aggregation processes, Kybernetika, 43(2):115-132, Institute of Information Theory and Automation Academy of Sciences of Czech Republic, 2007.

[9] J. Fodor, M. Roubens, Fuzzy Preference Modelling and Multicriteria Decision Support, Kluwer Acad. Publ., Dordrecht, 1994.

[10] S. Gottwald, A Treatise on Many-Valued Logics vol. 9: Studies in Logic and Computation, Research Studies Press, Baldock, Hertfordshire, England, 2001.

[11] O. Grigorenko, J. Lebedinska, On another view of aggregation of fuzzy relations, In S. Galichet, J. Montero and G. Mauris, editors, Proc. 7th Conf. EUSFLAT-2011 and LFA-2011, pages 21-27, Atlantis Press, 2011.

[12] E.P. Klement, R. Mesiar and E. Pap, Triangular Norms, Kluwer Acad. Publ., Dordrecht, 2000.

[13] R. Mesiar and S. Saminger, Domination of ordered weighted averaging operators over tnorms, Soft Computing, 8:562-570, Springer, 2004.

[14] S. Ovchinnikov, Similarity relations, fuzzy partitions, and fuzzy orderings, Fuzzy Sets Syst., 40:107-126, Elsevier, 1991.

[15] V. Peneva and I. Popchev, Properties of the aggregation operators related with fuzzy relations, Fuzzy Sets Syst., 139(3):615-633, Elsevier, 2003.

[16] M. Roubens, P. Vincke, Preference Modelling, Springer-Verlag, Berlin, 1985.

[17] S. Saminger, R. Mesiar and U. Bodenhofer, Domination of aggregation operators and preservation of transitivity, Internat. J. Uncertain., Fuzziness, Knowl.-Based Syst., 10(Suppl.):11-35, World Scientific, 2002.

[18] K. C. Maes, S. Saminger and B. De Baets, Representation and construction of self-dual aggregation operators, European Journal of Operational Research, 177:472-487, Elsevier, 2007.

[19] P. Sarkoci, Dominance is not transitive on continuous triangular norms, Aequationes Mathematicae, 75:201-207, Springer, 2008.

[20] L.A. Zadeh, Fuzzy sets, Inform. Control, 8:338-353, Elsevier, 1965.

[21] L.A. Zadeh, Similarity relations and fuzzy orderings, Inform. Sci., 3:177-200, Elsevier, 1971. 